\title{
Influence of Establishment Methods and Site-Specific Nitrogen Approaches on Growth Functions and Yield of Rice
}

\author{
M.R. Bhavya and M. Dinesh Kumar* \\ Department of Agronomy, College of Agriculture, University of Agricultural and \\ Horticultural Sciences, Shivamogga-577225, Karnataka, India \\ *Corresponding author
}

\section{Keywords}

Average growth rate, Dry matter, Growth functions, Leaf area, LAD, LCC and STCR

Article Info

Accepted: 26 April 2018 Available Online: 10 May 2018

\section{A B S T R A C T}

A Field experiment was taken up during kharif season of 2014 and 2015 on sandy loam soils at College of Agriculture, Navile, Shivamogga, Karnataka, India. Leaf area was significantly higher in the system of rice intensification (SRI) method of establishment from $30 \mathrm{DAT}$ and reached a peak value at 90 DAT then decreased. At 90 DAT, leaf area was $1717.21 \mathrm{~cm}^{2}$ as compared to aerobic $\left(967.97 \mathrm{~cm}^{2}\right)$ and conventional $\left(1002.31 \mathrm{~cm}^{2}\right)$ methods of establishment. The leaf area coverage in proportion to ground and also the functional ability of leaves remained more in SRI method resulting significantly higher total dry matter accumulation (116.03 $\mathrm{g} \mathrm{plant}^{-1}$ ) compared to conventional (95.73 $\mathrm{g} \mathrm{plant}^{-1}$ ) and aerobic (65.41 g plant $\left.{ }^{-1}\right)$ methods at harvest. As a result, it recorded significantly higher absolute $\left(0.63,0.84\right.$ and $1.60 \mathrm{~g} \mathrm{plant}^{-1} \mathrm{day}^{-1}$ at 30 to 60,60 to 90 and 90 DAT to harvest), relative $\left(0.028 \mathrm{gg}^{-1} \mathrm{day}^{-1}\right.$ up to $\left.90 \mathrm{DAT}\right)$ and crop growth rates. Thereby established higher grain $\left(7767 \mathrm{~kg} \mathrm{ha}^{-1}\right)$ and straw $\left(8878 \mathrm{~kg} \mathrm{ha}^{-1}\right)$ yield over other methods of establishments. Soil test and crop response (STCR) approach of nitrogen application recorded significantly higher leaf area $\left(1313.24 \mathrm{~cm}^{2}\right)$, leaf area index (3.20) and leaf area duration (111.87 days) at 90 days after sowing or transplanting closely followed by leaf colour chart (LCC) compared to other nitrogen management approaches. As a result, STCR approach recorded higher dry matter accumulation at all the growth stages $(4.08 \mathrm{~g}$ plant $^{-1}$ at 30 DAT, $29.73 \mathrm{~g} \mathrm{plant}^{-1}$ at 60 DAT, $57.82 \mathrm{~g} \mathrm{plant}^{-1}$ at 90 DAT and harvest it was $101.60 \mathrm{~g} \mathrm{plant}^{-1}$ ) along with absolute and relative growth rates resulting in significantly higher grain $\left(7183 \mathrm{~kg} \mathrm{ha}^{-1}\right)$ and straw yield $\left(8314 \mathrm{~kg} \mathrm{ha}^{-1}\right)$ closely followed by LCC approach over other nitrogen management approaches. Method of establishment by SRI with STCR approach of nitrogen management yielded better leaf area and its components to achieve higher yields $\left(8348 \mathrm{~kg} \mathrm{ha}^{-1}\right)$ and becomes best.

\section{Introduction}

Leaf area and dry matter are considered as most important parameters decide the performance of crop regarding the final economic product. Leaf area is important which focuses on photosynthetic activity and houses a source for photosynthate and these two are linearly related. In this regard, it is important that plant should possess optimum number of leaves and as well its greenery for autotrophic status. One of the most important growth indicators which have been considered as a measure of total photosynthesis and 
respiratory tissues is total dry weight. Total dry weight per se is increased over time with growth phase so that at early growth stages it increases with fewer gradients and at later stages, the slope of increase is greater as an indicator of the linear relationship until total dry weight reaches to its maximum (grain filling). The pre-requisite for getting higher yields in any crop is due to higher total dry matter production and it's partitioning into various plant parts coupled with the maximum translocation of photosynthates to the sink. The total dry matter production of a crop is an important parameter for determination of the performance of crop as it determines yield or economic part. Total dry matter accumulation also depends on many factors such as endogenous, environmental and cultural practices. However, light, radiation, humidity, soil moisture, availability of nutrients dictate both functionalities of leaf and as well the dry matter production at large.

Method of establishment has given prime importance in rice due to shortage of water for the traditional system of transplanting, paving the way for alternatives. In that SRI and aerobic system of establishment raised hopes without yield loss obtained to that of traditional practice. Hence, the methodology of adoption becomes crucial to harness the resources for getting higher leaf area and dry matter. Leaf functionality and photosynthesis are strongly influenced by the nutrient status, especially nitrogen in the cereal crops. Leaf area and its activity play a dominant role in photosynthesis and hence accumulation of carbohydrate and other photosynthates in the sink that are significantly influenced by orientation of crop and its management. Identification of physiological growth indices in the analysis of factors affecting yield and its components has great importance and its stability determines the dry matter production which is a criterion of yield and its components (Gardner et al., 1985). Hence in the study, an effort is made to document and explain the effect of methods of establishment and as well site-specific nitrogen management on different growth indices in bringing yield variations.

\section{Materials and Methods}

A field experiment was taken up during the kharif 2014 and 2015 at College of Agriculture, Navile, Shivamogga comes under Southern Transition Zone (Zone-7) of Karnataka. The geographical reference point of the experimental site was $13^{0} 58^{\prime}$ to $14^{0} 1^{\prime}$ North latitude and $75^{\circ} 34^{\prime}$ to $75^{\circ} 42^{\prime}$ East longitude with an altitude of $650 \mathrm{~m}$ above the mean sea level. The experiment was laid out in split plot design with three methods of establishment as main plots (aerobic, system of rice intensification and conventional) and nitrogen management approaches as subplots [Soil test and crop response (STCR), Soil test Based on lab (STL), Leaf colour chart (LCC) and Recommended fertilizers (RDF)], forming 12 treatment combinations with three replications. The main plots prepared by the desired environment/ecosystem wherein the subplots also were maintained under each main plot. The variety used in the experiment was KRH-4. Twelve days old seedlings were carefully planted (single seedling hill ${ }^{-1}$ ) at a spacing of $25 \times 25 \mathrm{~cm}$ in SRI system, two seeds per spot was dibbled at a spacing of $25 \mathrm{x}$ $25 \mathrm{~cm}$ accounting seed rate of five $\mathrm{kg} \mathrm{ha}^{-1}$. After ten days of sowing, only one seedling maintained by removing the excess seedling and necessary gaps was taken up during the time in case of aerobic system. Twenty-one day old seedlings were planted (one seedling hill $^{-1}$ ) at a spacing of $20 \mathrm{~cm} \mathrm{x} 15 \mathrm{~cm}$ in the conventional system.

A common dose of FYM @ 10 tonnes $\mathrm{ha}^{-1}$ was incorporated uniformly into the soil two weeks before planting for all the methods of establishment. For all the treatment plots, a 
common dose of $20 \mathrm{~kg} \quad \mathrm{ZnSO}_{4} \mathrm{ha}^{-1}$ was applied at the time of sowing/transplanting. The quantity of different major fertilizer used under different approaches mentioned below, for STCR approach under all the system of establishing the quantity of major plant nutrients was calculated with a target yield of $80 \mathrm{q} \mathrm{ha}^{-1}$ by using following target yield equations for Bhadra Command Area (Anon., 2008).

$\mathrm{FN}=2.981 \mathrm{~T}-0.30 \mathrm{SN}\left(\mathrm{KMnO}_{4}-\mathrm{N}\right)$

$\mathrm{FP}_{2} \mathrm{O}_{5}=1.232 \mathrm{~T}-0.786 \mathrm{SP}_{2} \mathrm{O}_{5}\left(\right.$ Bray's $\left.\mathrm{P}_{2} \mathrm{O}_{5}\right)$

$\mathrm{FK}_{2} \mathrm{O}=1.173 \mathrm{~T}-0.155 \mathrm{SK}_{2} \mathrm{O}\left(\mathrm{NH}_{4} \mathrm{OAC}-\right.$ $\mathrm{K}_{2} \mathrm{O}$ )

Where,

$\mathrm{T}=$ Targeted yield $\left(80 \mathrm{q} \mathrm{ha}^{-1}\right)$ i.e. $80 \mathrm{qha}^{-1}$

$\mathrm{FN}=$ Nitrogen supplied through Fertilizer $(\mathrm{kg}$ $\mathrm{ha}^{-1}$ )

$\mathrm{SN}=$ Initial available Nitrogen in soil $\left(\mathrm{kg} \mathrm{ha}^{-1}\right)$

$\mathrm{FP}_{2} \mathrm{O}_{5}=$ Phosphorous supplied through Fertilizer $\left(\mathrm{kg} \mathrm{ha}^{-1}\right)$

$\mathrm{SP}_{2} \mathrm{O}_{5}=$ Initial available $\mathrm{P}_{2} \mathrm{O}_{5}$ in soil $\left(\mathrm{kg} \mathrm{ha}^{-1}\right)$

$\mathrm{FK}_{2} \mathrm{O}=$ Potassium supplied through Fertilizer $\left(\mathrm{kg} \mathrm{ha}^{-1}\right)$

$\mathrm{SK}_{2} \mathrm{O}=$ Initial available $\mathrm{K}_{2} \mathrm{O}$ in soil $\left(\mathrm{kg} \mathrm{ha}^{-1}\right)$

Accordingly, the quantity of nitrogen was 175 and $176 \mathrm{~kg} \mathrm{ha}{ }^{1}$, wherein phosphorus and potassium levels stood at 55 and $56 \mathrm{~kg} \mathrm{ha}^{-1}$ in 2014 and 2015, respectively. In STL approach amount of fertilizer was calculated using soil test rating. Since for rice crop recommended dose of nitrogen is $100 \mathrm{~kg} \mathrm{ha}^{-1}$ and the soil of the experimental area was low in available nitrogen, using the properties of table, +12.5 $\mathrm{kg} \mathrm{ha}{ }^{-1}$ is added along with recommended dose. Considering their status for phosphorus and potassium no change is made in the level of application $\left(50 \mathrm{~kg} \mathrm{ha}^{-1}\right)$. Plots with leaf colour chart approach received a uniform dose of $14 \mathrm{~kg}$ nitrogen $\mathrm{ha}^{-1}$ as a basal dose further nitrogen is supplied to the crop based on LCC value of four and below (Balasubramanian et al., 1999). Readings started from 14 days in SRI and conventional methods after transplanting and from 21 days in the aerobic method at an interval of three days until first flowering. Nitrogen@ $25 \mathrm{~kg} \mathrm{ha}^{-1}$ was applied for SRI and conventional systems and $20 \mathrm{~kg}$ $\mathrm{ha}^{-1}$ was applied in the aerobic system at each LCC reading value of four and below. The total quantity of nitrogen used in the LCC based approach is 134 in case of aerobic and $164 \mathrm{~kg} \mathrm{ha}^{-1}$ in SRI and conventional systems in both the year of the experiment. The recommended dose of fertilizer is 100:50:50 $\mathrm{kg} \mathrm{N}: \mathrm{P}_{2} \mathrm{O}_{5}: \mathrm{K}_{2} \mathrm{O}$ ha $^{-1}$ as per the package of practice of 2010, University of Agricultural Sciences, Bangalore.

Other cultural practices by the package were taken up as per requirement of the crop. Leaf area and dry matter of the crop plants were taken at regular intervals. Various physiological parameters such as leaf area index (Watson, 1952), leaf area duration (Power et al., 1967), absolute growth rate (Radford, 1967), relative growth rate (Radford, 1967) and crop growth rate (Watson, 1952) were computed using standard formula available. Data so collected and computed were subjected to statistical analysis as per the design (Gomez and Gomez, 1984) for drawing meaningful inferences or conclusions.

\section{Results and Discussion}

In the present study, from initiation stage itself, SRI method of establishment produced higher leaf area throughout crop growth period 
envisaging its superiority. As seen from table 1 leaf area was significantly higher in SRI from 30 DAT and reached a peak at 90 DAT then decreased. At $90 \mathrm{DAT}$, leaf area was $1717.21 \mathrm{~cm}^{2}$ as compared to aerobic (967.97 $\left.\mathrm{cm}^{2}\right)$ and conventional $\left(1002.31 \mathrm{~cm}^{2}\right)$ methods of establishment. The wider spacing between plants under SRI management leads to more prolonged open crop canopy (Stoop, 2005; Thakur, 2010), truly contributing towards carbohydrate production (Lin et al., 2009). Hence, maintenance of leaf area till the end signifies bulk of photosynthate production and also translocation of the same towards biomass and sink (grain) till grain gets into physiological maturity. The positive effect of SRI method of establishment on leaf area is also noticed in the studies of Sowmyalatha (2011) and Suresh Naik (2014). Important growth indicators used as a photosynthetic system measurement is leaf area index and leaf area duration. The leaf area coverage in proportion to the ground, i.e. LAI (Table 2) and also the functional ability of leaves, i.e. LAD (Table 3) remained more in the treatment above and eventually stood better regarding performance. Stoop (2005) in his studies categorically mentioned higher LAI values for the traditional system $(20 \times 15 \mathrm{~cm})$ mainly because of population coverage per unit area. Leaf area index is related to the biologic and economic yields and increase in LAI causes higher yield (Singh et al., 2009). Leaf area index of rice plants planted at 16 plants $\mathrm{m}^{-2}$ was highest among other plant densities evaluated ( 7.5 or 19.5 plants $\mathrm{m}^{-2}$ ) at 20 days after heading (Lin et al., 2009).

In the present study, SRI method of establishment recorded significantly higher total dry matter (Table 4) accumulation (116.03 $\mathrm{g} \mathrm{plant}^{-1}$ ) compared to conventional (95.73 $\mathrm{g} \mathrm{plant}^{-1}$ )and aerobic (65.41 $\mathrm{g} \mathrm{plant}^{-1}$ ) methods at harvest (Table 4). Dry matter accumulation in individual plants depends on growth components and functional leaf area and SRI performed superior in these parameters indicating more of resource use efficiency like light and nutrients in a given situation followed by conventional methods of establishment. At all growth stages, dry matter portioning indicated the superiority of SRI over other methods of establishment. This work confirms the work of Pradeep Nayak (2009) and Suresh Naik (2014).

The computed parameters such as absolute growth rate (AGR), relative growth rate (RGR) and crop growth rate (CGR) by and large showed a similar tendency to that of dry matter accumulation (Table 5, 6 and 7 respectively). The rate of dry matter accumulation per day did differ with respect to different methods of establishment. Absolute growth rate was significantly higher under SRI method of establishment at all the stages $\left(0.63,0.84\right.$ and $1.60 \mathrm{~g} \mathrm{plant}^{-1} \mathrm{day}^{-1}$ at 30 to 60 , 60 to 90 and 90 DAT to harvest). Increased AGR was due to increase in the rate of dry accumulation per day. The first step in developing a procedure for analyzing dry weight changes was accounted by Blackman (1919). He pointed out that increase in dry weight regarded as a process of continuous compound interest, the increment produced in any interval adding to the capital for growth in subsequent periods. The rate of interest of dry matter accumulation (RGR) was significantly higher (0.028 $\mathrm{gg}^{-1} \mathrm{day}^{-1}$ up to 90 DAT) in SRI method of establishment. Crop growth rate has a direct relationship with photosynthetic surface and also LAI has a high impact on CGR before canopy closure. It is also noted that in the later stages of crop growth, i.e., 90 days onwards respectively higher value $(0.100$ $\mathrm{gm}^{-2} \mathrm{day}^{-1}$ ) of CGR in SRI truly validate the increase in translocation of photosynthate towards spikelet number. Horie et al., (2005) suggested based on their review of past scientific knowledge and their research data that a higher CGR at the late reproductive stage ( 2 weeks before heading) would be the 
most effective change for realizing higher yield. This concept implies that a high CGR can sustain an increase in spikelet numbers by controlling their degeneration together with high dry matter production which will contribute to boosting yield potential (Takai et al., 2006). SRI method of rice establishment achieved significantly higher grain $(7767 \mathrm{~kg}$ $\mathrm{ha}^{-1}$ ) and straw (8878 $\mathrm{kg} \mathrm{ha}^{-1}$ ) yield (Table 8). It recorded 56.11 and 49.26 per cent higher grain and straw yield over aerobic method (4975 and $5948 \mathrm{~kg} \mathrm{ha}^{-1}$ ), whereas it was 8.24 and 9.54 per cent over conventional method (7219 and $8105 \mathrm{~kg} \mathrm{ha}^{-1}$ ) of establishment.

Nitrogen strongly affects the plant growth and dry matter production. Different nitrogen management approaches significantly influenced the growth parameters of the rice crop. In general, different approaches succeeded in getting the timely progress of crop functional leaf area (Table 1). In any plant system, leaf area and its index (Table 2) reach a maximum just before flowering and decreases afterthat. In the present study, STCR approach recorded significantly higher leaf area $\left(1313.24 \mathrm{~cm}^{2}\right)$ and leaf area index (3.20) at $90 \mathrm{DAS} / \mathrm{T}$ closely followed by LCC as compared to other nitrogen management approaches (Table 4 and 5). Similarly, LAD (Table 3) also differed significantly due to nitrogen management approaches. STCR approach recorded significantly higher LAD (45.82, 102.94 and 111.87 days from 30 to 60, 60 to 90 and 90 to harvest, respectively) followed by LCC approach (41.90, 97.54 and 107.02 days from 30 to 60,60 to 90 and 90 to harvest, respectively) compared to other nitrogen approaches (Table 6). The increase in the growth parameters might be due to an adequate supply and timely availability of nitrogen to the rice plants during their early growth period helped for the initiation of leaves for its viable functionality over time. This helps in carbohydrate production and also timely cell division orienting towards increase in tallness, thereby maintains further growth without nutrient stress (Ramesh and Chandrasekaran, 2007; Kirk and Kronzucker, 2005). Nitrogen availability to that of crop largely determines these factors in a given environment (Anzoua et al., 2010). Leaf area index changing through alteration in nitrogen fertilizer levels is one of the most practical ways of variations (Mahdavi et al., 2006; Katsura et al., 2007).

This has reflected on accumulation of more photosynthate as could be seen from the data (Table 4) that STCR approach recorded higher dry matter accumulation at all the growth stages $\left(4.08 \mathrm{~g} \mathrm{plant}^{-1}\right.$ at $30 \mathrm{DAT}^{2} 29.73 \mathrm{~g}$ plant $^{-1}$ at 60 DAT, $57.82 \mathrm{~g} \mathrm{plant}^{-1}$ at 90 DAT and harvest it was $101.60 \mathrm{~g} \mathrm{plant}^{-1}$ ). Wherein other nitrogen management approaches like RDF $\left(3.02 \mathrm{~g} \mathrm{plant}^{-1}\right.$ at 30 DAT, $19.89 \mathrm{~g} \mathrm{plant}^{-1}$ at $60 \mathrm{DAT}, 48.64 \mathrm{~g} \mathrm{plant}^{-1}$ at $90 \mathrm{DAT}$ and $82.77 \mathrm{~g} \mathrm{ha}^{-1}$ at harvest), STL (3.34g plant ${ }^{-1}$ at 30 DAT, 23.76g plant ${ }^{-1}$ at 60 DAT, $52.48 \mathrm{~g}$ plant $^{-1}$ at 90 DAT and $90.18 \mathrm{~g} \mathrm{ha}^{-1}$ at harvest)

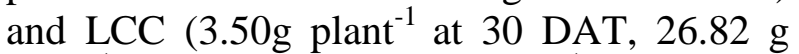
plant $^{-1}$ at $60 \mathrm{DAT}, 55.35 \mathrm{~g} \mathrm{plant}^{-1}$ at $90 \mathrm{DAT}$ and $95.03 \mathrm{~g} \mathrm{ha}^{-1}$ at harvest) recorded less. It envisages the transformation of carbohydrates from functional leaves very effectively in STCR and LCC approaches as compared to others.

It is evident from the data that total dry matter production varied significantly at different growth stages of crop due to the effect of different nitrogen management reflected on various growth and biomass accumulation (Prabhuraj et al., 2006). The trend of total dry matter production under nitrogen fertilizer contents reveals the fact that at initial there was a slow accumulation of photosynthate and after about 50 days, the changes were more noticeable. Maximum total dry matter accumulation at grain filling was more at higher nitrogen levels than lower ones (Fageria and Baligar, 2005; Fageria, 2007). 
Table.1 Leaf area of rice as influenced by systems of establishment and nitrogen management approaches at different growth stages

\begin{tabular}{|c|c|c|c|c|c|c|c|c|c|c|c|c|}
\hline \multirow[t]{3}{*}{ Treatments } & \multicolumn{12}{|c|}{ Leaf area $\left(\mathrm{cm}^{2}\right.$ plant $\left.{ }^{-1}\right)$} \\
\hline & \multicolumn{3}{|c|}{$30 \mathrm{DAS} / \mathrm{T}$} & \multicolumn{3}{|c|}{$60 \mathrm{DAS} / \mathrm{T}$} & \multicolumn{3}{|c|}{90 DAS/T } & \multicolumn{3}{|c|}{ At harvest } \\
\hline & 2014 & 2015 & Pooled & 2014 & 2015 & Pooled & 2014 & 2015 & Pooled & 2014 & 2015 & Pooled \\
\hline \multicolumn{13}{|c|}{ Methods of establishment (S) } \\
\hline $\mathbf{S}_{1}-$ Aerobic & 103.39 & 104.97 & 104.18 & 514.82 & 550.30 & 532.56 & 984.60 & 951.34 & 967.97 & 794.30 & 917.73 & 856.01 \\
\hline $\mathbf{S}_{2}-\mathbf{S R I}$ & 227.65 & 284.23 & 255.94 & 1113.63 & 1198.28 & 1155.95 & 1686.76 & 1747.66 & 1717.21 & 963.40 & 906.50 & 934.95 \\
\hline $\mathbf{S}_{3}-$ Conventional & 168.53 & 237.03 & 202.78 & 829.63 & 1008.28 & 918.96 & 983.12 & 1021.51 & 1002.31 & 848.70 & 808.11 & 828.40 \\
\hline S.Em \pm & 3.61 & 7.81 & 4.30 & 35.33 & 14.33 & 19.07 & 17.59 & 52.86 & 27.85 & 23.08 & 19.33 & 15.05 \\
\hline C.D. $(p=0.05)$ & 14.17 & 30.65 & 14.02 & 138.74 & 56.27 & 62.18 & 69.07 & 207.54 & 90.84 & 90.60 & 75.90 & 49.08 \\
\hline \multicolumn{13}{|c|}{ Nitrogen management approaches $(\mathbf{N})$} \\
\hline $\mathbf{N}_{1}-\mathrm{STCR}$ & 205.47 & 230.63 & 218.05 & 890.57 & 1001.75 & 946.16 & 1301.40 & 1325.07 & 1313.24 & 956.00 & 936.93 & 946.46 \\
\hline $\mathbf{N}_{2}-\mathbf{S T L}$ & 158.82 & 194.77 & 176.79 & 805.20 & 882.82 & 844.01 & 1203.98 & 1214.45 & 1209.22 & 861.05 & 845.73 & 853.39 \\
\hline $\mathbf{N}_{3}-\mathbf{L C C}$ & 165.19 & 214.80 & 190.00 & 824.52 & 949.92 & 887.22 & 1255.29 & 1248.85 & 1252.07 & 888.82 & 917.34 & 903.08 \\
\hline $\mathbf{N}_{4}-\mathbf{R D F}$ & 136.61 & 194.78 & 165.69 & 757.15 & 841.33 & 799.24 & 1111.96 & 1172.31 & 1142.13 & 769.33 & 809.79 & 789.56 \\
\hline S.Em \pm & 7.34 & 9.69 & 6.08 & 28.58 & 27.95 & 19.99 & 33.94 & 36.24 & 24.83 & 32.28 & 22.72 & 19.74 \\
\hline C.D. $(p=0.05)$ & 21.80 & 28.79 & 17.43 & 84.92 & 83.04 & 57.33 & 100.85 & 107.67 & 71.21 & 95.92 & 67.52 & 56.62 \\
\hline \multicolumn{13}{|l|}{ Interaction (SxN) } \\
\hline $\mathrm{S}_{1} \mathbf{N}_{1}$ & 121.10 & 122.93 & 122.01 & 571.90 & 607.63 & 589.76 & 1047.13 & 986.34 & 1016.73 & 827.01 & 988.28 & 907.64 \\
\hline $\mathbf{S}_{1} \mathbf{N}_{2}$ & 98.89 & 99.73 & 99.31 & 495.26 & 530.66 & 512.96 & 972.62 & 941.90 & 957.26 & 796.13 & 898.07 & 847.10 \\
\hline $\mathbf{S}_{1} \mathbf{N}_{3}$ & 110.58 & 112.41 & 111.49 & 533.30 & 568.70 & 551.00 & 998.01 & 953.94 & 975.98 & 805.25 & 967.18 & 886.21 \\
\hline $\mathrm{S}_{1} \mathbf{N}_{4}$ & 82.98 & 84.81 & 83.90 & 458.81 & 494.21 & 476.51 & 920.63 & 923.19 & 921.91 & 748.80 & 817.40 & 783.10 \\
\hline $\mathbf{S}_{2} \mathbf{N}_{1}$ & 271.90 & 307.07 & 289.49 & 1224.15 & 1326.55 & 1275.35 & 1834.39 & 1873.63 & 1854.01 & 1138.29 & 973.56 & 1055.93 \\
\hline $\mathbf{S}_{2} \mathbf{N}_{2}$ & 202.05 & 253.89 & 227.97 & 1084.96 & 1120.36 & 1102.66 & 1659.26 & 1715.16 & 1687.21 & 950.28 & 853.54 & 901.91 \\
\hline $\mathrm{S}_{2} \mathrm{~N}_{3}$ & 233.04 & 284.87 & 258.95 & 1102.08 & 1267.48 & 1184.78 & 1765.81 & 1768.35 & 1767.08 & 986.84 & 952.12 & 969.48 \\
\hline $\mathbf{S}_{2} \mathbf{N}_{4}$ & 203.61 & 291.11 & 247.36 & 1043.33 & 1078.73 & 1061.03 & 1487.59 & 1633.49 & 1560.54 & 778.19 & 846.79 & 812.49 \\
\hline $\mathbf{S}_{3} \mathbf{N}_{1}$ & 223.40 & 261.90 & 242.65 & 875.65 & 1071.05 & 973.35 & 1022.69 & 1115.26 & 1068.97 & 902.69 & 848.95 & 875.82 \\
\hline $\mathrm{S}_{3} \mathbf{N}_{2}$ & 175.52 & 230.69 & 203.10 & 835.38 & 997.45 & 916.41 & 980.07 & 986.30 & 983.19 & 836.74 & 785.57 & 811.15 \\
\hline $\mathbf{S}_{3} \mathbf{N}_{3}$ & 151.96 & 247.13 & 199.55 & 838.19 & 1013.59 & 925.89 & 1002.05 & 1024.25 & 1013.15 & 874.38 & 832.72 & 853.55 \\
\hline $\mathbf{S}_{3} \mathbf{N}_{4}$ & 123.24 & 208.41 & 165.82 & 769.31 & 951.04 & 860.18 & 927.67 & 960.24 & 943.95 & 781.00 & 765.19 & 773.09 \\
\hline S.Em \pm & 12.71 & 16.78 & 10.53 & 49.51 & 48.41 & 34.62 & 58.79 & 62.77 & 43.00 & 55.92 & 39.36 & 34.19 \\
\hline C.D. $(p=0.05)$ & NS & NS & NS & NS & NS & NS & NS & NS & NS & NS & NS & NS \\
\hline CV $(\%)$ & 13.22 & 13.93 & 13.74 & 10.47 & 9.12 & 9.76 & 8.36 & 8.77 & 8.57 & 11.15 & 7.77 & 9.59 \\
\hline
\end{tabular}

DAS/T: Days after sowing/ transplanting, NS: Non-Significant 
Table.2 Leaf area index of rice as influenced by systems of establishment and nitrogen management approaches at different growth stages

\begin{tabular}{|c|c|c|c|c|c|c|c|c|c|c|c|c|}
\hline \multirow[t]{3}{*}{ Treatments } & \multicolumn{12}{|c|}{ Leaf area index (LAI) } \\
\hline & \multicolumn{3}{|c|}{$30 \mathrm{DAS} / \mathrm{T}$} & \multicolumn{3}{|c|}{$60 \mathrm{DAS} / \mathrm{T}$} & \multicolumn{3}{|c|}{$90 \mathrm{DAS} / \mathrm{T}$} & \multicolumn{3}{|c|}{ At harvest } \\
\hline & 2014 & 2015 & Pooled & 2014 & 2015 & Pooled & 2014 & 2015 & Pooled & 2014 & 2015 & Pooled \\
\hline \multicolumn{13}{|c|}{ Methods of establishment (S) } \\
\hline $\mathbf{S}_{1}-$ Aerobic & 0.16 & 0.17 & 0.17 & 0.82 & 0.88 & 0.85 & 1.53 & 1.52 & 1.53 & 1.27 & 1.47 & 1.37 \\
\hline $\mathbf{S}_{2}-\mathrm{SRI}$ & 0.36 & 0.45 & 0.41 & 1.78 & 1.92 & 1.85 & 3.38 & 2.80 & 3.09 & 1.54 & 1.53 & 1.54 \\
\hline $\mathbf{S}_{3}-$ Conventional & 0.56 & 0.79 & 0.67 & 2.77 & 3.36 & 3.06 & 5.02 & 3.41 & 4.21 & 2.83 & 2.69 & 2.76 \\
\hline S.Em \pm & 0.02 & 0.02 & 0.01 & 0.06 & 0.03 & 0.03 & 0.24 & 0.08 & 0.12 & 0.03 & 0.04 & 0.03 \\
\hline C.D. $(p=0.05)$ & 0.09 & 0.06 & 0.05 & 0.23 & 0.12 & 0.11 & 0.93 & 0.31 & 0.41 & 0.13 & 0.17 & 0.09 \\
\hline \multicolumn{13}{|c|}{ Nitrogen management approaches $(\mathbf{N})$} \\
\hline $\mathbf{N}_{1}-\mathrm{STCR}$ & 0.46 & 0.52 & 0.49 & 1.93 & 2.22 & 2.08 & 3.63 & 2.76 & 3.20 & 2.05 & 2.03 & 2.04 \\
\hline $\mathbf{N}_{2}-$ STL & 0.35 & 0.44 & 0.40 & 1.77 & 1.99 & 1.88 & 3.23 & 2.51 & 2.87 & 1.86 & 1.84 & 1.85 \\
\hline $\mathbf{N}_{3}-\mathbf{L C C}$ & 0.35 & 0.49 & 0.42 & 1.80 & 2.11 & 1.95 & 3.45 & 2.59 & 3.02 & 1.93 & 1.97 & 1.95 \\
\hline $\mathbf{N}_{4}-\mathrm{RDF}$ & 0.29 & 0.43 & 0.36 & 1.66 & 1.90 & 1.78 & 2.93 & 2.43 & 2.68 & 1.68 & 1.76 & 1.72 \\
\hline S.Em \pm & 0.03 & 0.02 & 0.02 & 0.06 & 0.05 & 0.04 & 0.16 & 0.06 & 0.08 & 0.06 & 0.05 & 0.04 \\
\hline C.D. $(p=0.05)$ & 0.10 & 0.06 & 0.05 & 0.17 & 0.16 & 0.11 & 0.46 & 0.18 & 0.24 & 0.18 & 0.16 & 0.11 \\
\hline \multicolumn{13}{|l|}{ Interaction (SxN) } \\
\hline $\mathrm{S}_{1} \mathrm{~N}_{1}$ & 0.19 & 0.20 & 0.20 & 0.92 & 0.97 & 0.94 & 1.46 & 1.58 & 1.52 & 1.32 & 1.58 & 1.45 \\
\hline $\mathrm{S}_{1} \mathbf{N}_{2}$ & 0.15 & 0.16 & 0.16 & 0.79 & 0.85 & 0.82 & 1.56 & 1.51 & 1.53 & 1.27 & 1.44 & 1.36 \\
\hline $\mathbf{S}_{1} \mathbf{N}_{3}$ & 0.18 & 0.18 & 0.18 & 0.85 & 0.91 & 0.88 & 1.58 & 1.53 & 1.55 & 1.29 & 1.55 & 1.42 \\
\hline $\mathrm{S}_{\mathbf{1}} \mathbf{N}_{4}$ & 0.13 & 0.14 & 0.13 & 0.73 & 0.79 & 0.76 & 1.53 & 1.48 & 1.50 & 1.20 & 1.31 & 1.25 \\
\hline $\mathbf{S}_{2} \mathbf{N}_{1}$ & 0.44 & 0.49 & 0.46 & 1.96 & 2.12 & 2.04 & 3.84 & 3.00 & 3.42 & 1.82 & 1.66 & 1.74 \\
\hline $\mathbf{S}_{2} \mathbf{N}_{2}$ & 0.32 & 0.41 & 0.36 & 1.74 & 1.79 & 1.76 & 3.29 & 2.74 & 3.02 & 1.52 & 1.47 & 1.50 \\
\hline $\mathrm{S}_{2} \mathrm{~N}_{3}$ & 0.37 & 0.46 & 0.41 & 1.76 & 2.03 & 1.90 & 3.47 & 2.83 & 3.15 & 1.58 & 1.58 & 1.58 \\
\hline $\mathbf{S}_{2} \mathbf{N}_{4}$ & 0.33 & 0.47 & 0.40 & 1.67 & 1.73 & 1.70 & 2.91 & 2.61 & 2.76 & 1.25 & 1.41 & 1.33 \\
\hline $\mathbf{S}_{3} \mathbf{N}_{1}$ & 0.74 & 0.87 & 0.81 & 2.92 & 3.57 & 3.24 & 5.60 & 3.72 & 4.66 & 3.01 & 2.83 & 2.92 \\
\hline $\mathrm{S}_{3} \mathrm{~N}_{2}$ & 0.57 & 0.77 & 0.67 & 2.78 & 3.32 & 3.05 & 4.83 & 3.29 & 4.06 & 2.79 & 2.62 & 2.70 \\
\hline $\mathbf{S}_{3} \mathbf{N}_{3}$ & 0.51 & 0.82 & 0.67 & 2.79 & 3.38 & 3.09 & 5.30 & 3.41 & 4.36 & 2.91 & 2.78 & 2.85 \\
\hline$\overline{\mathbf{S}_{3} \mathbf{N}_{4}}$ & 0.41 & 0.69 & 0.55 & 2.56 & 3.17 & 2.87 & 4.36 & 3.20 & 3.78 & 2.60 & 2.55 & 2.58 \\
\hline S.Em \pm & 0.06 & 0.03 & 0.03 & 0.10 & 0.09 & 0.07 & 0.27 & 0.11 & 0.15 & 0.10 & 0.09 & 0.07 \\
\hline C.D. $(p=0.05)$ & NS & NS & NS & NS & NS & NS & NS & NS & NS & NS & NS & NS \\
\hline CV (\%) & 27.10 & 12.58 & 19.46 & 9.43 & 7.92 & 8.62 & 14.11 & 7.22 & 12.08 & 9.59 & 8.38 & 9.00 \\
\hline
\end{tabular}


Table.3 Leaf area duration of rice as influenced by systems of establishment and nitrogen management approaches at different growth stages

\begin{tabular}{|c|c|c|c|c|c|c|c|c|c|}
\hline \multirow[t]{3}{*}{ Treatments } & \multicolumn{9}{|c|}{ Leaf area duration (Days) } \\
\hline & \multicolumn{3}{|c|}{30 - $60 \mathrm{DAS} / \mathrm{T}$} & \multicolumn{3}{|c|}{60 - 90 DAS/T } & \multicolumn{3}{|c|}{90 DAS/T - At harvest } \\
\hline & 2014 & 2015 & Pooled & 2014 & 2015 & Pooled & 2014 & 2015 & Pooled \\
\hline \multicolumn{10}{|c|}{ Methods of establishment (S) } \\
\hline $\mathbf{S}_{1}-$ Aerobic & 17.28 & 18.25 & 17.76 & 48.14 & 49.25 & 48.69 & 65.92 & 67.69 & 66.81 \\
\hline $\mathbf{S}_{2}-\mathbf{S R I}$ & 37.65 & 42.40 & 40.03 & 93.94 & 99.46 & 96.70 & 104.09 & 106.84 & 105.46 \\
\hline$S_{3}-$ Conventional & 58.25 & 74.12 & 66.18 & 132.23 & 151.90 & 142.07 & 140.97 & 142.56 & 141.76 \\
\hline S.Em \pm & 0.89 & 0.63 & 0.54 & 2.00 & 1.66 & 1.30 & 1.04 & 2.78 & 1.48 \\
\hline C.D. $(p=0.05)$ & 3.48 & 2.49 & 1.78 & 7.84 & 6.53 & 4.24 & 4.06 & 10.91 & 4.84 \\
\hline \multicolumn{10}{|c|}{ Nitrogen management approaches $(\mathbf{N})$} \\
\hline $\mathrm{N}_{1}-\mathrm{STCR}$ & 42.70 & 48.93 & 45.82 & 97.76 & 108.12 & 102.94 & 110.43 & 113.31 & 111.87 \\
\hline $\mathbf{N}_{2}-\mathbf{S T L}$ & 37.07 & 43.18 & 40.12 & 90.52 & 97.36 & 93.94 & 102.70 & 103.03 & 102.86 \\
\hline $\mathbf{N}_{3}-\mathbf{L C C}$ & 37.62 & 46.18 & 41.90 & 93.07 & 102.01 & 97.54 & 106.83 & 107.20 & 107.02 \\
\hline $\mathbf{N}_{4}-\mathrm{RDF}$ & 33.53 & 41.40 & 37.46 & 84.40 & 93.33 & 88.87 & 94.69 & 99.25 & 96.97 \\
\hline S.Em \pm & 1.25 & 1.04 & 0.81 & 1.81 & 2.19 & 1.42 & 1.65 & 2.13 & 1.34 \\
\hline C.D. $(p=0.05)$ & 3.72 & 3.08 & 2.33 & 5.38 & 6.51 & 4.08 & 4.90 & 6.32 & 3.86 \\
\hline \multicolumn{10}{|l|}{ Interaction (SxN) } \\
\hline $\mathbf{S}_{1} \mathbf{N}_{1}$ & 19.54 & 20.48 & 20.01 & 51.78 & 52.84 & 52.31 & 68.51 & 71.06 & 69.79 \\
\hline $\mathbf{S}_{1} \mathbf{N}_{2}$ & 16.47 & 17.52 & 17.00 & 47.12 & 48.08 & 47.60 & 65.79 & 66.76 & 66.28 \\
\hline $\mathbf{S}_{1} \mathbf{N}_{3}$ & 18.11 & 19.04 & 18.58 & 49.55 & 50.19 & 49.87 & 67.23 & 69.00 & 68.12 \\
\hline $\mathbf{S}_{1} \mathbf{N}_{4}$ & 14.99 & 15.93 & 15.46 & 44.12 & 45.88 & 45.00 & 62.16 & 63.93 & 63.05 \\
\hline $\mathbf{S}_{2} \mathbf{N}_{1}$ & 42.43 & 46.58 & 44.50 & 102.78 & 108.64 & 105.71 & 115.37 & 114.90 & 115.13 \\
\hline $\mathbf{S}_{2} \mathbf{N}_{2}$ & 35.74 & 39.08 & 37.41 & 91.90 & 94.94 & 93.42 & 102.45 & 104.41 & 103.43 \\
\hline $\mathbf{S}_{2} \mathbf{N}_{3}$ & 37.64 & 44.09 & 40.86 & 95.28 & 103.28 & 99.28 & 108.44 & 108.53 & 108.49 \\
\hline $\mathbf{S}_{2} \mathbf{N}_{4}$ & 34.81 & 39.86 & 37.34 & 85.78 & 90.98 & 88.38 & 90.08 & 99.53 & 94.81 \\
\hline$\overline{\mathbf{S}_{3} \mathbf{N}_{1}}$ & 66.12 & 79.74 & 72.93 & 138.70 & 162.87 & 150.78 & 147.40 & 153.97 & 150.69 \\
\hline $\mathbf{S}_{3} \mathbf{N}_{2}$ & 58.99 & 72.94 & 65.96 & 132.54 & 149.06 & 140.80 & 139.84 & 137.91 & 138.88 \\
\hline $\mathbf{S}_{3} \mathbf{N}_{3}$ & 57.11 & 75.39 & 66.25 & 134.37 & 152.57 & 143.47 & 144.82 & 144.06 & 144.44 \\
\hline $\mathbf{S}_{3} \mathbf{N}_{4}$ & 50.79 & 68.39 & 59.59 & 123.31 & 143.12 & 133.22 & 131.82 & 134.28 & 133.05 \\
\hline S.Em \pm & 2.17 & 1.80 & 1.41 & 3.13 & 3.80 & 2.46 & 2.85 & 3.68 & 2.33 \\
\hline C.D. $(p=0.05)$ & NS & NS & NS & NS & NS & NS & NS & NS & NS \\
\hline CV $(\%)$ & 9.95 & 6.92 & 8.34 & 5.94 & 6.56 & 6.29 & 4.77 & 6.03 & 5.45 \\
\hline
\end{tabular}


Table.4 Total dry matter accumulation of rice as influenced by systems of establishment and nitrogen management approaches

\begin{tabular}{|c|c|c|c|c|c|c|c|c|c|c|c|c|}
\hline \multirow[t]{3}{*}{ Treatments } & \multicolumn{12}{|c|}{ Total dry matter accumulation (g plant ${ }^{-1}$ ) } \\
\hline & \multicolumn{3}{|c|}{$30 \mathrm{DAS} / \mathrm{T}$} & \multicolumn{3}{|c|}{$60 \mathrm{DAS} / \mathrm{T}$} & \multicolumn{3}{|c|}{90 DAS/T } & \multicolumn{3}{|c|}{ At harvest } \\
\hline & 2014 & 2015 & Pooled & 2014 & 2015 & Pooled & 2014 & 2015 & Pooled & 2014 & 2015 & Pooled \\
\hline \multicolumn{13}{|c|}{ Methods of establishment (S) } \\
\hline $\mathbf{S}_{1}-$ Aerobic & 1.88 & 1.93 & 1.90 & 13.04 & 12.88 & 12.96 & 36.54 & 37.09 & 36.82 & 65.53 & 65.29 & 65.41 \\
\hline $\mathbf{S}_{2}-\mathbf{S R I}$ & 5.27 & 5.37 & 5.32 & 33.69 & 35.92 & 34.80 & 65.55 & 65.55 & 65.55 & 115.28 & 116.78 & 116.03 \\
\hline$S_{3}-$ Conventional & 3.19 & 3.27 & 3.23 & 27.44 & 27.34 & 27.39 & 58.34 & 58.35 & 58.35 & 94.84 & 96.63 & 95.73 \\
\hline S.Em \pm & 0.14 & 0.18 & 0.11 & 1.10 & 1.38 & 0.88 & 1.08 & 0.71 & 0.65 & 4.21 & 4.49 & 3.08 \\
\hline C.D. $(p=0.05)$ & 0.54 & 0.72 & 0.37 & 4.31 & 5.44 & 2.88 & 4.23 & 2.78 & 2.10 & 16.55 & 17.62 & 10.04 \\
\hline \multicolumn{13}{|c|}{ Nitrogen management approaches $(\mathbf{N})$} \\
\hline $\mathrm{N}_{1}-\mathrm{STCR}$ & 4.04 & 4.12 & 4.08 & 29.38 & 30.09 & 29.73 & 57.85 & 57.78 & 57.82 & 102.96 & 100.23 & 101.60 \\
\hline $\mathbf{N}_{2}-\mathbf{S T L}$ & 3.31 & 3.37 & 3.34 & 23.03 & 24.49 & 23.76 & 52.38 & 52.58 & 52.48 & 90.09 & 90.28 & 90.18 \\
\hline $\mathbf{N}_{3}-\mathbf{L C C}$ & 3.45 & 3.55 & 3.50 & 26.70 & 26.93 & 26.82 & 55.48 & 55.23 & 55.35 & 94.55 & 95.50 & 95.03 \\
\hline $\mathbf{N}_{4}-\mathrm{RDF}$ & 2.98 & 3.06 & 3.02 & 19.77 & 20.00 & 19.89 & 48.21 & 49.07 & 48.64 & 79.94 & 85.59 & 82.77 \\
\hline S.Em \pm & 0.19 & 0.13 & 0.12 & 0.68 & 0.85 & 0.54 & 1.77 & 1.29 & 1.09 & 3.06 & 4.06 & 2.54 \\
\hline C.D. $(p=0.05)$ & 0.56 & 0.40 & 0.33 & 2.01 & 2.54 & 1.56 & 5.25 & 3.83 & 3.13 & 9.10 & NS & 7.29 \\
\hline \multicolumn{13}{|l|}{ Interaction(SxN) } \\
\hline $\mathbf{S}_{1} \mathbf{N}_{1}$ & 2.04 & 2.05 & 2.05 & 15.34 & 15.66 & 15.50 & 39.58 & 40.46 & 40.02 & 75.30 & 73.04 & 74.17 \\
\hline $\mathbf{S}_{1} \mathbf{N}_{2}$ & 1.88 & 1.93 & 1.90 & 11.30 & 11.32 & 11.31 & 36.20 & 36.40 & 36.30 & 64.55 & 63.58 & 64.07 \\
\hline $\mathrm{S}_{1} \mathbf{N}_{3}$ & 1.90 & 1.98 & 1.94 & 14.13 & 13.71 & 13.92 & 37.89 & 38.15 & 38.02 & 65.68 & 65.26 & 65.47 \\
\hline $\mathrm{S}_{1} \mathbf{N}_{4}$ & 1.69 & 1.76 & 1.72 & 11.38 & 10.83 & 11.11 & 32.49 & 33.36 & 32.93 & 56.59 & 59.30 & 57.95 \\
\hline $\mathbf{S}_{2} \mathbf{N}_{1}$ & 6.39 & 6.54 & 6.46 & 41.45 & 43.67 & 42.56 & 70.91 & 69.31 & 70.11 & 127.55 & 124.26 & 125.91 \\
\hline $\mathbf{S}_{2} \mathbf{N}_{2}$ & 4.92 & 4.94 & 4.93 & 31.77 & 36.21 & 33.99 & 64.31 & 64.51 & 64.41 & 112.89 & 113.73 & 113.31 \\
\hline $\mathbf{S}_{2} \mathbf{N}_{3}$ & 5.13 & 5.28 & 5.21 & 37.54 & 38.46 & 38.00 & 67.06 & 66.60 & 66.83 & 118.95 & 120.52 & 119.73 \\
\hline $\mathbf{S}_{2} \mathbf{N}_{4}$ & 4.64 & 4.72 & 4.68 & 24.00 & 25.32 & 24.66 & 59.92 & 61.79 & 60.85 & 101.73 & 108.62 & 105.18 \\
\hline $\mathrm{S}_{3} \mathbf{N}_{1}$ & 3.69 & 3.77 & 3.73 & 31.34 & 30.94 & 31.14 & 63.05 & 63.58 & 63.32 & 106.02 & 103.40 & 104.71 \\
\hline $\mathrm{S}_{3} \mathbf{N}_{2}$ & 3.13 & 3.24 & 3.19 & 26.03 & 25.93 & 25.98 & 56.62 & 56.82 & 56.72 & 92.82 & 93.52 & 93.17 \\
\hline $\mathrm{S}_{3} \mathbf{N}_{3}$ & 3.31 & 3.39 & 3.35 & 28.44 & 28.62 & 28.53 & 61.48 & 60.93 & 61.20 & 99.03 & 100.74 & 99.88 \\
\hline $\mathrm{S}_{3} \mathbf{N}_{4}$ & 2.61 & 2.69 & 2.65 & 23.94 & 23.86 & 23.90 & 52.21 & 52.07 & 52.14 & 81.48 & 88.86 & 85.17 \\
\hline S.Em \pm & 0.33 & 0.23 & 0.20 & 1.17 & 1.48 & 0.94 & 3.06 & 2.23 & 1.89 & 5.31 & 7.03 & 4.40 \\
\hline C.D. $(p=0.05)$ & NS & NS & 0.57 & 3.48 & 4.39 & 2.71 & NS & NS & NS & NS & NS & NS \\
\hline CV (\%) & 16.37 & 11.36 & 14.03 & 8.21 & 10.09 & 9.22 & 9.91 & 7.20 & 8.65 & 10.00 & 13.10 & 11.67 \\
\hline
\end{tabular}

DAS/T: Days after sowing/ transplanting, NS: Non-Significant 
Table.5 Absolute growth rate of rice as influenced by systems of establishment and nitrogen management approaches at different growth stages

\begin{tabular}{|c|c|c|c|c|c|c|c|c|c|}
\hline \multirow[t]{3}{*}{ Treatments } & \multicolumn{9}{|c|}{ Absolute growth rate $\left(\mathrm{g} \mathrm{day}^{-1}\right)$} \\
\hline & \multicolumn{3}{|c|}{30 - 60 DAS/T } & \multicolumn{3}{|c|}{60 - 90 DAS/T } & \multicolumn{3}{|c|}{90 DAS/T - At harvest } \\
\hline & 2014 & 2015 & Pooled & 2014 & 2015 & Pooled & 2014 & 2015 & Pooled \\
\hline \multicolumn{10}{|c|}{ Methods of establishment (S) } \\
\hline $\mathbf{S}_{1}-$ Aerobic & 0.22 & 0.23 & 0.23 & 0.67 & 0.67 & 0.67 & 0.83 & 0.82 & 0.83 \\
\hline $\mathbf{S}_{2}-\mathrm{SRI}$ & 0.62 & 0.64 & 0.63 & 0.86 & 0.83 & 0.84 & 1.62 & 1.59 & 1.60 \\
\hline$S_{3}-$ Conventional & 0.53 & 0.54 & 0.53 & 0.82 & 0.80 & 0.81 & 1.21 & 1.16 & 1.18 \\
\hline S.Em \pm & 0.03 & 0.02 & 0.02 & 0.04 & 0.03 & 0.03 & 0.13 & 0.10 & 0.08 \\
\hline C.D. $(p=0.05)$ & 0.11 & 0.10 & 0.06 & NS & NS & 0.09 & 0.51 & 0.40 & 0.27 \\
\hline \multicolumn{10}{|c|}{ Nitrogen management approaches $(\mathbf{N})$} \\
\hline $\mathbf{N}_{1}-\mathrm{STCR}$ & 0.53 & 0.54 & 0.53 & 0.82 & 0.79 & 0.80 & 1.42 & 1.42 & 1.42 \\
\hline $\mathbf{N}_{2}-\mathrm{STL}$ & 0.44 & 0.45 & 0.44 & 0.79 & 0.77 & 0.78 & 1.18 & 1.18 & 1.18 \\
\hline $\mathbf{N _ { 3 } - \mathbf { L C C }}$ & 0.50 & 0.51 & 0.50 & 0.80 & 0.77 & 0.79 & 1.25 & 1.24 & 1.24 \\
\hline$\overline{\mathbf{N}_{4}-\mathbf{R D F}}$ & 0.37 & 0.38 & 0.38 & 0.73 & 0.74 & 0.74 & 1.02 & 0.93 & 0.97 \\
\hline S.Em \pm & 0.03 & 0.03 & 0.02 & 0.06 & 0.04 & 0.04 & 0.12 & 0.09 & 0.08 \\
\hline C.D. $(p=0.05)$ & 0.08 & 0.08 & 0.06 & NS & NS & NS & NS & 0.27 & 0.22 \\
\hline \multicolumn{10}{|l|}{ Interaction (SxN) } \\
\hline $\mathbf{S}_{1} \mathbf{N}_{1}$ & 0.25 & 0.26 & 0.26 & 0.72 & 0.72 & 0.72 & 1.01 & 1.04 & 1.03 \\
\hline $\mathbf{S}_{1} \mathbf{N}_{2}$ & 0.19 & 0.21 & 0.20 & 0.71 & 0.68 & 0.69 & 0.81 & 0.81 & 0.81 \\
\hline $\mathbf{S}_{1} \mathbf{N}_{3}$ & 0.25 & 0.26 & 0.25 & 0.68 & 0.67 & 0.68 & 0.80 & 0.82 & 0.81 \\
\hline$\overline{S_{1} \mathbf{N}_{4}}$ & 0.19 & 0.20 & 0.20 & 0.60 & 0.61 & 0.60 & 0.69 & 0.63 & 0.66 \\
\hline $\mathrm{S}_{2} \mathrm{~N}_{1}$ & 0.77 & 0.79 & 0.78 & 0.83 & 0.75 & 0.79 & 1.83 & 1.83 & 1.83 \\
\hline $\mathbf{S}_{2} \mathbf{N}_{2}$ & 0.60 & 0.61 & 0.60 & 0.86 & 0.85 & 0.86 & 1.58 & 1.56 & 1.57 \\
\hline $\mathbf{S}_{2} \mathbf{N}_{3}$ & 0.70 & 0.71 & 0.71 & 0.83 & 0.80 & 0.81 & 1.70 & 1.72 & 1.71 \\
\hline $\mathbf{S}_{2} \mathbf{N}_{4}$ & 0.43 & 0.44 & 0.44 & 0.90 & 0.94 & 0.92 & 1.37 & 1.24 & 1.31 \\
\hline $\mathbf{S}_{3} \mathbf{N}_{1}$ & 0.56 & 0.56 & 0.56 & 0.90 & 0.90 & 0.90 & 1.41 & 1.39 & 1.40 \\
\hline $\mathbf{S}_{3} \mathbf{N}_{2}$ & 0.52 & 0.53 & 0.53 & 0.79 & 0.78 & 0.79 & 1.16 & 1.18 & 1.17 \\
\hline$\overline{\mathbf{S}_{3} \mathbf{N}_{3}}$ & 0.54 & 0.55 & 0.54 & 0.89 & 0.85 & 0.87 & 1.26 & 1.18 & 1.22 \\
\hline $\mathbf{S}_{3} \mathbf{N}_{4}$ & 0.50 & 0.51 & 0.50 & 0.71 & 0.69 & 0.70 & 1.00 & 0.91 & 0.95 \\
\hline S.Em \pm & 0.05 & 0.05 & 0.03 & 0.10 & 0.07 & 0.06 & 0.21 & 0.16 & 0.13 \\
\hline C.D. $(p=0.05)$ & NS & NS & 0.10 & NS & NS & NS & NS & NS & NS \\
\hline CV $(\%)$ & 18.00 & 17.34 & 17.67 & 22.89 & 15.57 & 19.65 & 29.24 & 23.09 & 26.41 \\
\hline
\end{tabular}


Table.6 Relative growth rate of rice as influenced by systems of establishment and nitrogen management approaches at different growth stages

\begin{tabular}{|c|c|c|c|c|c|c|c|c|c|}
\hline \multirow[t]{3}{*}{ Treatments } & \multicolumn{9}{|c|}{ Relative growth rate $\left(\mathrm{g} \mathrm{g}^{-1}\right.$ day $\left.^{-1}\right)$} \\
\hline & \multicolumn{3}{|c|}{30 - $60 \mathrm{DAS} / \mathrm{T}$} & \multicolumn{3}{|c|}{60 - 90 DAS/T } & \multicolumn{3}{|c|}{90 DAS/T - At harvest } \\
\hline & 2014 & 2015 & Pooled & 2014 & 2015 & Pooled & 2014 & 2015 & Pooled \\
\hline \multicolumn{10}{|c|}{ Methods of establishment (S) } \\
\hline $\mathbf{S}_{1}-$ Aerobic & 0.054 & 0.055 & 0.054 & 0.042 & 0.040 & 0.041 & 0.020 & 0.020 & 0.020 \\
\hline $\mathbf{S}_{2}-\mathrm{SRI}$ & 0.064 & 0.064 & 0.064 & 0.027 & 0.026 & 0.026 & 0.023 & 0.023 & 0.023 \\
\hline$S_{3}-$ Conventional & 0.071 & 0.071 & 0.071 & 0.029 & 0.028 & 0.028 & 0.021 & 0.020 & 0.020 \\
\hline S.Em & 0.003 & 0.002 & 0.002 & 0.003 & 0.001 & 0.002 & 0.002 & 0.002 & 0.001 \\
\hline C.D. $(p=0.05)$ & 0.011 & 0.008 & 0.006 & 0.011 & 0.006 & 0.005 & NS & NS & NS \\
\hline \multicolumn{10}{|c|}{ Nitrogen management approaches $(\mathbf{N})$} \\
\hline $\mathbf{N}_{1}-\mathbf{S T C R}$ & 0.064 & 0.064 & 0.064 & 0.030 & 0.029 & 0.030 & 0.023 & 0.023 & 0.023 \\
\hline $\mathbf{N}_{2}-\mathbf{S T L}$ & 0.061 & 0.062 & 0.062 & 0.034 & 0.033 & 0.033 & 0.021 & 0.021 & 0.021 \\
\hline $\mathbf{N}_{3}-\mathrm{LCC}$ & 0.065 & 0.065 & 0.065 & 0.031 & 0.030 & 0.031 & 0.021 & 0.021 & 0.021 \\
\hline $\mathbf{N}_{4}-\mathrm{RDF}$ & 0.061 & 0.061 & 0.061 & 0.034 & 0.034 & 0.034 & 0.021 & 0.019 & 0.020 \\
\hline S.Em \pm & 0.003 & 0.002 & 0.002 & 0.002 & 0.002 & 0.001 & 0.002 & 0.001 & 0.001 \\
\hline C.D. $(p=0.05)$ & NS & NS & NS & NS & NS & NS & NS & NS & NS \\
\hline \multicolumn{10}{|l|}{ Interaction (SxN) } \\
\hline$\overline{\mathbf{S}_{1} \mathbf{N}_{1}}$ & 0.057 & 0.057 & 0.057 & 0.040 & 0.039 & 0.039 & 0.023 & 0.023 & 0.023 \\
\hline $\mathbf{S}_{1} \mathbf{N}_{2}$ & 0.048 & 0.052 & 0.050 & 0.047 & 0.043 & 0.045 & 0.020 & 0.020 & 0.020 \\
\hline$\overline{\mathbf{S}_{1} \mathbf{N}_{3}}$ & 0.057 & 0.057 & 0.057 & 0.040 & 0.039 & 0.039 & 0.019 & 0.019 & 0.019 \\
\hline $\mathbf{S}_{1} \mathbf{N}_{4}$ & 0.053 & 0.053 & 0.053 & 0.042 & 0.041 & 0.041 & 0.020 & 0.019 & 0.019 \\
\hline$\overline{\mathbf{S}_{2} \mathbf{N}_{1}}$ & 0.065 & 0.065 & 0.065 & 0.022 & 0.020 & 0.021 & 0.024 & 0.025 & 0.025 \\
\hline $\mathrm{S}_{2} \mathbf{N}_{2}$ & 0.065 & 0.065 & 0.065 & 0.027 & 0.027 & 0.027 & 0.023 & 0.023 & 0.023 \\
\hline $\mathbf{S}_{2} \mathbf{N}_{3}$ & 0.068 & 0.068 & 0.068 & 0.024 & 0.023 & 0.023 & 0.024 & 0.024 & 0.024 \\
\hline $\mathbf{S}_{2} \mathbf{N}_{4}$ & 0.057 & 0.057 & 0.057 & 0.034 & 0.034 & 0.034 & 0.022 & 0.020 & 0.021 \\
\hline $\mathbf{S}_{3} \mathbf{N}_{1}$ & 0.070 & 0.069 & 0.069 & 0.029 & 0.029 & 0.029 & 0.023 & 0.021 & 0.022 \\
\hline $\mathbf{S}_{3} \mathbf{N}_{2}$ & 0.070 & 0.070 & 0.070 & 0.029 & 0.028 & 0.029 & 0.020 & 0.021 & 0.020 \\
\hline $\mathbf{S}_{3} \mathbf{N}_{3}$ & 0.071 & 0.071 & 0.071 & 0.030 & 0.029 & 0.029 & 0.020 & 0.020 & 0.020 \\
\hline $\mathbf{S}_{3} \mathbf{N}_{4}$ & 0.073 & 0.073 & 0.073 & 0.027 & 0.026 & 0.027 & 0.019 & 0.018 & 0.019 \\
\hline S.Em \pm & 0.005 & 0.004 & 0.003 & 0.004 & 0.003 & 0.002 & 0.003 & 0.002 & 0.002 \\
\hline C.D. $(p=0.05)$ & NS & NS & NS & NS & NS & 0.007 & NS & NS & NS \\
\hline CV (\%) & 12.56 & 9.69 & 11.21 & 19.41 & 15.81 & 17.77 & 25.19 & 19.89 & 22.71 \\
\hline
\end{tabular}


Table.7 Crop growth rate of rice as influenced by systems of establishment and nitrogen management approaches at different growth stages

\begin{tabular}{|c|c|c|c|c|c|c|c|c|c|}
\hline \multirow[t]{3}{*}{ Treatments } & \multicolumn{9}{|c|}{ Crop growth rate $\left(\mathrm{g} \mathrm{m}^{-2}\right.$ day $\left.^{-1}\right)$} \\
\hline & \multicolumn{3}{|c|}{30 - $60 \mathrm{DAS} / \mathrm{T}$} & \multicolumn{3}{|c|}{60 - 90 DAS/T } & \multicolumn{3}{|c|}{90 DAS/T - At harvest } \\
\hline & 2014 & 2015 & Pooled & 2014 & 2015 & Pooled & 2014 & 2015 & Pooled \\
\hline \multicolumn{10}{|c|}{ Methods of establishment (S) } \\
\hline $\mathbf{S}_{1}-$ Aerobic & 0.014 & 0.015 & 0.014 & 0.042 & 0.042 & 0.042 & 0.052 & 0.052 & 0.052 \\
\hline $\mathbf{S}_{2}-\mathrm{SRI}$ & 0.039 & 0.040 & 0.039 & 0.054 & 0.052 & 0.053 & 0.101 & 0.099 & 0.100 \\
\hline$S_{3}-$ Conventional & 0.016 & 0.016 & 0.016 & 0.025 & 0.024 & 0.025 & 0.037 & 0.035 & 0.036 \\
\hline S.Em & 0.001 & 0.001 & 0.001 & 0.002 & 0.002 & 0.001 & 0.007 & 0.006 & 0.005 \\
\hline C.D. $(p=0.05)$ & 0.006 & 0.004 & 0.003 & 0.006 & 0.008 & 0.004 & 0.028 & 0.024 & 0.015 \\
\hline \multicolumn{10}{|c|}{ Nitrogen management approaches $(\mathbf{N})$} \\
\hline $\mathbf{N}_{1}-\mathbf{S T C R}$ & 0.027 & 0.027 & 0.027 & 0.041 & 0.040 & 0.040 & 0.074 & 0.074 & 0.074 \\
\hline $\mathbf{N}_{2}-\mathbf{S T L}$ & 0.022 & 0.022 & 0.022 & 0.041 & 0.040 & 0.040 & 0.061 & 0.061 & 0.061 \\
\hline $\mathbf{N}_{3}-\mathrm{LCC}$ & 0.025 & 0.026 & 0.025 & 0.040 & 0.039 & 0.040 & 0.065 & 0.065 & 0.065 \\
\hline $\mathbf{N}_{4}-\mathrm{RDF}$ & 0.018 & 0.019 & 0.018 & 0.038 & 0.039 & 0.039 & 0.053 & 0.048 & 0.051 \\
\hline S.Em \pm & 0.001 & 0.001 & 0.001 & 0.002 & 0.002 & 0.002 & 0.007 & 0.006 & 0.004 \\
\hline C.D. $(p=0.05)$ & 0.004 & 0.004 & 0.003 & NS & NS & NS & NS & 0.017 & 0.013 \\
\hline \multicolumn{10}{|l|}{ Interaction (SxN) } \\
\hline$\overline{\mathbf{S}_{1} \mathbf{N}_{1}}$ & 0.016 & 0.016 & 0.016 & 0.045 & 0.045 & 0.045 & 0.063 & 0.065 & 0.064 \\
\hline $\mathbf{S}_{1} \mathbf{N}_{2}$ & 0.012 & 0.013 & 0.012 & 0.044 & 0.043 & 0.043 & 0.050 & 0.050 & 0.050 \\
\hline$\overline{\mathbf{S}_{1} \mathbf{N}_{3}}$ & 0.016 & 0.016 & 0.016 & 0.043 & 0.042 & 0.042 & 0.050 & 0.051 & 0.051 \\
\hline $\mathbf{S}_{1} \mathbf{N}_{4}$ & 0.012 & 0.013 & 0.012 & 0.037 & 0.038 & 0.038 & 0.043 & 0.039 & 0.041 \\
\hline$\overline{\mathbf{S}_{2} \mathbf{N}_{1}}$ & 0.048 & 0.049 & 0.048 & 0.052 & 0.047 & 0.049 & 0.115 & 0.115 & 0.115 \\
\hline $\mathrm{S}_{2} \mathbf{N}_{2}$ & 0.037 & 0.038 & 0.038 & 0.054 & 0.053 & 0.054 & 0.099 & 0.097 & 0.098 \\
\hline $\mathbf{S}_{2} \mathbf{N}_{3}$ & 0.044 & 0.044 & 0.044 & 0.052 & 0.050 & 0.051 & 0.106 & 0.107 & 0.107 \\
\hline $\mathbf{S}_{2} \mathbf{N}_{4}$ & 0.027 & 0.028 & 0.027 & 0.056 & 0.059 & 0.057 & 0.086 & 0.078 & 0.082 \\
\hline $\mathbf{S}_{3} \mathbf{N}_{1}$ & 0.017 & 0.017 & 0.017 & 0.027 & 0.027 & 0.027 & 0.043 & 0.042 & 0.042 \\
\hline $\mathbf{S}_{3} \mathbf{N}_{2}$ & 0.016 & 0.016 & 0.016 & 0.024 & 0.024 & 0.024 & 0.035 & 0.036 & 0.035 \\
\hline $\mathbf{S}_{3} \mathbf{N}_{3}$ & 0.016 & 0.017 & 0.017 & 0.027 & 0.026 & 0.026 & 0.038 & 0.036 & 0.037 \\
\hline $\mathbf{S}_{3} \mathbf{N}_{4}$ & 0.015 & 0.015 & 0.015 & 0.021 & 0.021 & 0.021 & 0.030 & 0.028 & 0.029 \\
\hline S.Em \pm & 0.002 & 0.003 & 0.002 & 0.004 & 0.004 & 0.003 & 0.012 & 0.010 & 0.008 \\
\hline C.D. $(p=0.05)$ & 0.007 & 0.007 & 0.005 & NS & NS & NS & NS & NS & NS \\
\hline CV (\%) & 18.17 & 18.40 & 18.29 & 17.13 & 17.90 & 17.52 & 32.70 & 27.05 & 30.06 \\
\hline
\end{tabular}


Table.8 Grain yield, straw yield, harvest index and test weight of rice as influenced by systems of establishment and nitrogen management approaches

\begin{tabular}{|c|c|c|c|c|c|c|}
\hline \multirow[t]{2}{*}{ Treatments } & \multicolumn{3}{|c|}{ Grain yield $\left(\mathrm{kg} \mathrm{ha}^{-1}\right)$} & \multicolumn{3}{|c|}{ Straw yield $\left(\mathrm{kg} \mathrm{ha}^{-1}\right)$} \\
\hline & 2014 & 2015 & Pooled & 2014 & 2015 & Pooled \\
\hline \multicolumn{7}{|c|}{ Method of Establishment (S) } \\
\hline $\mathbf{S}_{1}-$ Aerobic & 4933 & 5018 & 4975 & 5948 & 5949 & 5948 \\
\hline $\mathbf{S}_{2}-\mathbf{S R I}$ & 7726 & 7807 & 7767 & 8882 & 8875 & 8879 \\
\hline $\mathbf{S}_{3}-$ Conventional & 7132 & 7219 & 7175 & 8177 & 8032 & 8105 \\
\hline S.Em \pm & 78 & 89 & 59 & 296 & 275 & 202 \\
\hline C.D. $(p=0.05)$ & 307 & 351 & 194 & 1164 & 1079 & 659 \\
\hline \multicolumn{7}{|c|}{ Nitrogen Management Approaches $(\mathbf{N})$} \\
\hline $\mathbf{N}_{1}-\mathrm{STCR}$ & 7144 & 7222 & 7183 & 8362 & 8266 & 8314 \\
\hline $\mathbf{N}_{2}-\mathbf{S T L}$ & 6353 & 6433 & 6393 & 7584 & 7593 & 7588 \\
\hline $\mathbf{N}_{3}-\mathbf{L C C}$ & 6735 & 6835 & 6785 & 7687 & 7707 & 7697 \\
\hline $\mathbf{N}_{4}-\mathbf{R D F}$ & 6156 & 6237 & 6196 & 7044 & 6909 & 6977 \\
\hline S.Em \pm & 182 & 192 & 132 & 303 & 313 & 218 \\
\hline C.D. $(p=0.05)$ & 540 & 570 & 379 & 900 & 929 & 625 \\
\hline \multicolumn{7}{|l|}{ Interaction (S X N) } \\
\hline $\mathbf{S}_{1} \mathbf{N}_{1}$ & 5242 & 5314 & 5278 & 6481 & 6490 & 6486 \\
\hline $\mathbf{S}_{1} \mathbf{N}_{2}$ & 4794 & 4872 & 4833 & 5894 & 5903 & 5899 \\
\hline $\mathbf{S}_{1} \mathbf{N}_{3}$ & 5014 & 5128 & 5071 & 5939 & 5948 & 5943 \\
\hline $\mathbf{S}_{1} \mathbf{N}_{4}$ & 4678 & 4759 & 4719 & 5478 & 5454 & 5466 \\
\hline $\mathrm{S}_{2} \mathbf{N}_{1}$ & 8308 & 8389 & 8348 & 9474 & 9483 & 9479 \\
\hline $\mathrm{S}_{2} \mathbf{N}_{2}$ & 7477 & 7558 & 7517 & 8870 & 8879 & 8875 \\
\hline $\mathbf{S}_{2} \mathbf{N}_{3}$ & 7874 & 7954 & 7914 & 8939 & 8982 & 8960 \\
\hline $\mathbf{S}_{2} \mathbf{N}_{4}$ & 7248 & 7329 & 7288 & 8246 & 8155 & 8201 \\
\hline $\mathbf{S}_{3} \mathbf{N}_{1}$ & 7881 & 7962 & 7921 & 9131 & 8824 & 8977 \\
\hline $\mathrm{S}_{3} \mathbf{N}_{2}$ & 6787 & 6868 & 6828 & 7987 & 7996 & 7992 \\
\hline $\mathbf{S}_{3} \mathbf{N}_{3}$ & 7317 & 7421 & 7369 & 8183 & 8192 & 8188 \\
\hline $\mathbf{S}_{3} \mathbf{N}_{4}$ & 6542 & 6623 & 6582 & 7408 & 7117 & 7263 \\
\hline S.Em \pm & 315 & 332 & 229 & 525 & 542 & 378 \\
\hline C.D. $(p=0.05)$ & NS & NS & NS & NS & NS & NS \\
\hline CV (\%) & 8.26 & 8.61 & 8.44 & 11.85 & 12.32 & 12.09 \\
\hline
\end{tabular}


Based on dry matter accumulation, various growth ancillary functions are computed such as AGR, RGR and CGR (Table 5, 6 and 7 respectively). All these growth functions more or less followed the trend to that of dry matter. The dry matter accumulation per day steadily increased throughout crop cycle from $0.53 \mathrm{~g} \mathrm{plant}^{-1} \mathrm{day}^{-1}$ for 30 to $60 \mathrm{DAS} / \mathrm{T}$ to later stages of 90 to at harvest $1.42 \mathrm{~g}$ plant $^{-}$ ${ }^{1}$ day $^{-1}$ in STCR followed by LCC approach compared to other nitrogen management approaches. The findings supported by the studies of Takai et al., (2006). Further dry matter accumulation per day over accumulated dry weight like compound interest also favored by STCR and LCC approach wherein the timely availability of nutrients with that of the plant need to be coincided to make this significant difference Zhang et al., (2007). Different nitrogen management approaches did not influence significantly on CGR and NAR as the integral differences were narrowed down. This might be due to integrated effect of maximum leaf area, photosynthesis, carbohydrate accumulation and timely transportation (Horie et al., 2005). Since leaf area is both an expression of size and partial expression of photosynthetic potential, the growth rate can be the multiple of two events such as NAR and mean leaf area, increase in the leaf/ NAR promote the dry matter production (Takai et al., 2006; Azarpour et al., 2011). Significantly higher grain $\left(7183 \mathrm{~kg} \mathrm{ha}^{-1}\right)$ and straw yield (8314 $\mathrm{kg} \mathrm{ha}^{-1}$ ) under STCR approach (Table 8) is mainly attributed due to positive and significant effect of the above factors. This was closely followed by LCC approach (6785 $\mathrm{kg}$ grain yield $\mathrm{ha}^{-1}$ ) though both systems performed well, but higher grain panicle in STCR made the approach excel. Variation in the straw yield due to the treatment effect followed the same trend as that of yield variation. Higher the straw yield under STCR approach is mainly due to variation in the dry matter accumulation.
It is interesting to note that in different methods of establishment tried, $\mathrm{N}$ management with STCR performed better followed by LCC approach. However, yield variations do exist among the interaction treatments. Highest grain yield was obtained in SRI method of establishment with STCR approach (8348 $\left.\mathrm{kg} \mathrm{ha}^{-1}\right)$. It was closely followed by SRI method of establishment with LCC (7914 $\mathrm{kg} \mathrm{ha}^{-1}$ ) or conventional methods of establishment with STCR (7922 $\mathrm{kg} \mathrm{ha}{ }^{-1}$ ) approach of $\mathrm{N}$ management (Table 8). The better performance under SRI method is mainly due to combination of transplanting early aged (12-14 days old), single seedlings per hill, shallow depth of planting with wider spacing $(25 \times 25 \mathrm{~cm})$, least injury to the roots, quick establishment promoting deeper and better distribution of root systems. It paved way for improved uptake of nitrogen obtained from STCR approach helped to obtain more total tillers plant ${ }^{-1}$ thus capitalizing comparatively better in terms of different growth parameters, leaf area $\left(1854.01 \mathrm{~cm}^{2}\right)$ and dry matter accumulation (125.91 g plant $^{-1}$ ) followed by its growth computed parameters. The result obtained strengthens the findings of Sowmyalatha (2011) and Singh et al., (2015).

The results of the present investigation permit to infer that SRI method of establishment with STCR approach of nitrogen management becomes best. SRI method of establishment with LCC approach or conventional system of establishment with STCR approach of nitrogen management becomes the second option.

\section{References}

Anonymous, 2008. Annual progress report, AICRP on soil test crop response correlation UAS, Bangalore, p. 27.

Anzoua, K.G., Junichi, K., Toshihiro, H., Kazuto, I. and Yutaka, J. 2010. Genetic 
improvements for high yield and low soil nitrogen tolerance in rice (Oryza sativa L.) under a cold environment. Field Crops Res. 116: 38-45.

Azarpour, E., Motamed, M.K., Moraditochaee, M. and Bozorgi, H.R. 2011. Effect of nitrogen fertilizer and nitrox in biofertilizer management on growth analysis and yield of rice cultivars (Iran). World Applied Sci. J. 14(2):193-198.

Balasubramanian, V. Morales, A.C., Cruz, R.T. and Abdulraehman, 1999. On-farm adoption of knowledge-intensive nitrogen management technologies for rice system. Nutrient Cycling Agroecosystem. 53:59-69.

Blackman, V.H. 1919. The compound interest law and plant growth. Annals of Botany. 33: 353-360.

Fageria, N.K. 2007. Yield physiology of rice. J. Plant Nutr. 30: 843-879.

Fageria, N.K. and Baligar, V.C., 2005. Enhancing nitrogen use efficiency in crop plants. Adv. Agron. 88: 97-185.

Gardner, F., Pearce, R. and Mitchell, R.L. 1985. Physiology of crop plants. Iowa state University Press. Ames. USA.

Gomez, K.A. and Gomez, A.A. 1984. Statistical procedures for agricultural research. $2^{\text {nd }}$ Edition. John Willey and Sons, New York.

Horie, T., Shiraiwa, T., Homma, K., Katsura, K., Maeda, S. and Yoshida, H. 2005. Can yields of low land rice resume the increases that they showed in the1980s? Plant Prod. Sci. 8: 259-274.

Katsura, K., Maeda, S., Horie, T. and Shiraiwa, T. 2007. Analysis of yield attributes and crop physiological traits of Liangyoupeijiu, hybrid rice recently bred in China. Field Crops Res.103: 170-177.

Kirk, G.J.D. and Kronzucker, H.J. 2005. The potential for nitrification and nitrate uptake in the rhizosphere of wetland plants: A modeling study. Annals of Botany, 96: 639-646.

Lin, X.Q., Zhu, D.F., Chen, H.Z., Cheng, S.H. and Uphoff, N. 2009. Effect of plant density and nitrogen fertilizer rates on grain yield and nitrogen uptake of hybrid rice (Oryza sativa L.). J. Agric. Biochem. Sust. Develop. 1: 44-53.

Mahdavi, F., Esmaeili, M.A., Fallah, A. and Pirdashti, H. 2006. Study of morphological characteristics, physiological indices, grain yield and its components in rice (Oryza sativa L.) landraces and improved cultivar. Iranian J. Crop Sci. 7(4): 280-297.

Power, J.F., Willis, W.O., Gunes, D.L. and Peichman, G.A. 1967. Effect of soil temperature, phosphorus and plant age growth on growth analysis of Barley. Agronomy Journal. 59: 231-234.

Prabhuraj, D.K., Thimmareddy, H., Mahadevappa, L. and Bongale, U.D. 2006. Soil test based fertilizer requirement for specific yield target of M5 mulberry in Alfisol under irrigated conditions. Mysore J. Agric. Sci. 40(4): 464-470

Pradeep Nayak, 2009. Studies on improvement of water productivity for rice (Oryza sativa L.) production through different methods of establishment, M.Sc. (Agri.) Thesis, Uni. Agric. Sci. Bangalore, India.

Radfors, P.J. 1967. Growth analysis formula: their use and abuse. Crop science. 8:171-175.

Ramesh, S. and Chandrasekaran, B. 2007. Effect of establishment techniques and nitrogen management on LNC, flowering, nitrogen use efficiency and quality of rice hybrid. Indian J. Agron. 2(1): 38-45.

Singh, A.K., Gautam, U.S., Singh, J., Singh, A. and Shrivastava, P. 2015. Impact of nutrient management technologies in transplanted rice under irrigated 
domains of Central India. African $\mathrm{J}$. Agric. Res. 10(5): 345-350.

Singh, D.K., Singh, J.K. and Singh, L. 2009. Real-time nitrogen management for higher $\mathrm{N}$ use efficiency in transplanted rice (Oryza sativa L.) under temperate Kashmir conditions. Indian J. Agric. Sci. 79(10): 772-775.

Sowmyalatha, B.S. 2011. Evaluation of rice hybrids under different methods of cultivation and fertility levels in Cauvery command area. M. Sc. (Agri.) Thesis, Univ. Agric. Sci. Bangalore, India.

Stoop, W.A. and Kassam, A.H. 2005. The SRI controversy: A response. Field Crops Res. 91: 357-360.

Suresh Naik, K.P. 2014. Studies on agronomic practices to mitigate methane emission under different methods of rice (Oryza sativa L.) cultivation. Ph. D. Thesis, Univ. Agric. Sci. Bangalore, India.

Takai, T., Matsuura, S., Nishio, T., Ohsumi, A., Shiraiwa, T. and Horie, T. 2006. Rice yield potential is closely related to crop growth rate during late reproductive period. Field Crops Res. 96: 328-335.

Thakur, A.K. 2010. SRI criticism: Beyond skepticism with empiricism. Current Sci. 98: 1294-1299.

Whatson, D.J. 1952. The physiological basis off variation in yield. Advances of Agronomy. 4:101-145.

Zhang, Q.F. 2007. Strategies for developing green super rice. In: Proc. National academy science united states America, 104: 16402-16409.

\section{How to cite this article:}

Bhavya, M.R. and Dinesh Kumar, M. 2018. Influence of Establishment Methods and SiteSpecific Nitrogen Approaches on Growth Functions and Yield of Rice. Int.J.Curr.Microbiol.App.Sci. 7(05): 3627-3642. doi: https://doi.org/10.20546/ijcmas.2018.705.419 